\title{
Compact broadband planar orthomode transducer
}

\author{
P.K. Grimes ${ }^{\star}$, O.G. King, G. Yassin and M.E. Jones \\ Dept. of Physics, University of Oxford, Denys Wilkinson Building, Keble Road, Oxford OX1 3RH, UK
}

25 October 2018

\begin{abstract}
We present the design and test results of a compact C-band orthomode transducer which comprises four rectangular probes orthogonally arranged in a circular waveguide, designed to work in the WG13 band. Measurements of the system in the frequency range $4.64 \mathrm{GHz}$ to $7.05 \mathrm{GHz}$ agree very well with simulation results and show a cross-polarisation level below $-58 \mathrm{~dB}$, a return loss of about $-20 \mathrm{~dB}$, and an insertion loss difference of less than $0.18 \mathrm{~dB}$ between the orthogonal polarisation modes across the full waveguide band.
\end{abstract}

\section{INTRODUCTION}

An orthomode transducer (OMT) is used to extract two orthogonal polarisation modes from a rectangular or circular waveguide. Several radio and mm cosmological experiments are now being designed to measure weakly polarised sources with an unprecedented sensitivity and accuracy (Taylor 2006). These instruments require an OMT with extremely low cross-polarisation of order $-50 \mathrm{~dB}$ or less and near-equal insertion loss of both orthogonal modes over a relatively wide band. The OMT must also be compact, easy to fabricate and integrate with planar circuits, and coolable to $4.2 \mathrm{~K}$.

An L-band OMT design, comprising four probes at right angles in a cylindrical waveguide, proposed by D. Bock (Bock 1999) has many of these required properties. Two orthogonal polarisations are extracted by combining the signals from each pair of opposite probes. It is compact, simple to construct, intrinsically relatively wideband, and easy to scale to any frequency band. Attempts to realise a high performance OMT using this design have so far fallen short of achieving the stringent requirements of new polarisation cosmology instruments, either because the bandwidth was too narrow (Jackson 2001) or the cross-polarisation was too high (Engargiola and Plambeck 2003).

The OMT described in this paper was developed for the C-Band All-Sky Survey (C-BASS) receiver which is being constructed by the Experimental Radio Cosmology group (ERC) at Oxford. The C-BASS receiver will be used to produce an all-sky map with unprecedented accuracy and sensitivity of the C-band polarised synchrotron radiation from our Galaxy. The data will be used primarily in the subtraction of foregrounds from upcoming measurements of

\footnotetext{
^ Email: pxg@astro.ox.ac.uk
}

This paper is a preprint of a paper accepted by Electronics Letters and is subject to Institution of Engineering and Technology Copyright. When the final version is published, the copy of record will be available at IET Digital Library
Figure 1. Top view of the completed OMT. The waveguide diameter is $41.4 \mathrm{~mm}$, the probe dimensions are $11.46 \mathrm{~mm}$ by $4.25 \mathrm{~mm}$, and the backshort length is $14.8 \mathrm{~mm}$

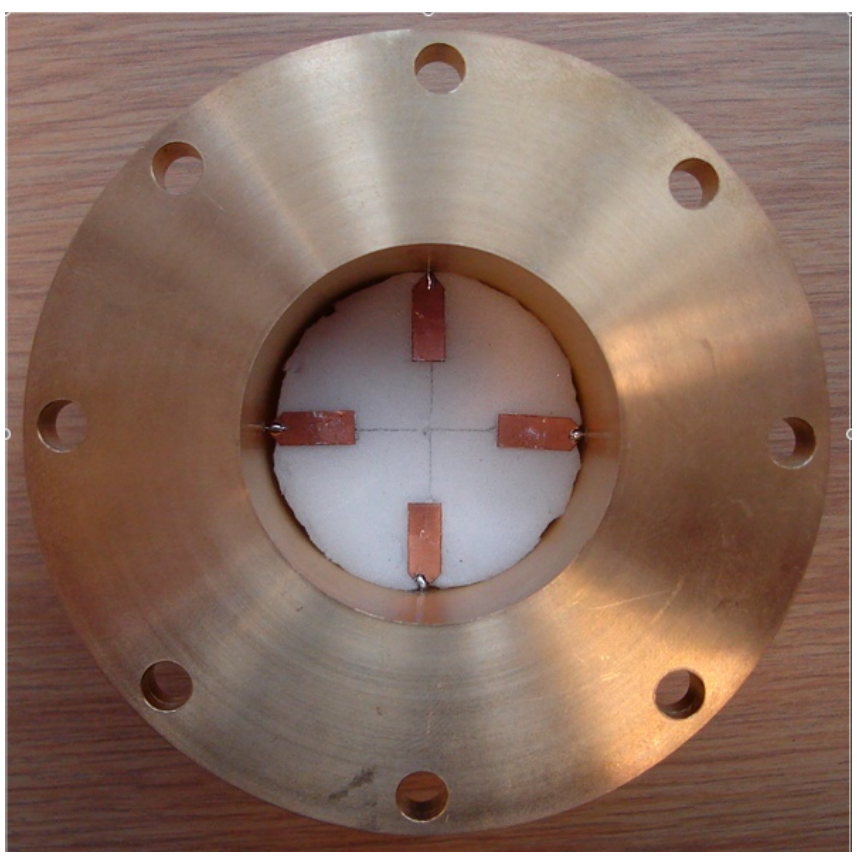

the B-mode polarised component of the Cosmic Microwave Background (CMB) (Taylor 2006).

\section{DESIGN AND SIMULATION}

The OMT was designed to cover the full WG13 $(4.64 \mathrm{GHz}$ to $7.05 \mathrm{GHz}$ ) band. The waveguide body was machined from brass and contained four rectangular probes, cut from $0.2 \mathrm{~mm}$ thick copper sheeting, placed orthogonally in a single cross-sectional plane of a circular waveguide on a supporting bed of a very low dielectric constant substance $(\simeq 1.06$, Plas- 
tazote $\mathrm{LD} 45^{1}$, which filled the space between the probes and the backshort (Fig. 1). The probes were tapered at an angle of $45^{\circ}$ to the waveguide walls to reduce the capacitance and were soldered to the pins of the SMA jacks, which were grounded to the body of the waveguide and fed through the wall in front of a fixed backshort. The signals from each pair of probes were combined by equal-length semi-rigid cables to a $180^{\circ}$ hybrid, based on a two branch rat-race design (Knöchel and Mayer 1990), to produce the in-phase orthogonal polarisation signals.

The OMT was modeled with Ansoft's HFS: ${ }^{2}$ package using thin perfectly conducting probes and $50 \Omega$ lumped element ports, with the probe dimensions and backshort length optimised for good return loss over a $45 \%$ bandwidth. The optimum OMT waveguide model was combined with the hybrid model in Ansoft Designer ${ }^{3}$ to produce simulations of the full OMT.

\section{EXPERIMENTAL RESULTS}

The system was tested using an Anritsu 37369C Vector Network Analyser (VNA), calibrated to remove the effects of the coaxial cables only. The OMT measurement setup is shown in Fig. 2 Port 1 of the VNA was connected to a coax-torectangular waveguide adaptor. This fed a rectangular-tocircular waveguide transition and then a circular waveguide section which was connected to the OMT input. Each polarisation component was measured by terminating the outputs from one pair of opposite probes while the second pair of opposite probes were connected to the hybrid. Port 2 of the VNA was connected to the difference output of the hybrid and the sum output of the hybrid was terminated with a matched load. The circular waveguide section was added to cut off any spurious evanescent cross-polarised modes produced by the rectangular-to-circular transition.

The insertion loss and return loss of each pair of probes were measured by aligning the input waveguide with the target probes. The cross-polarisation for each pair of probes was then measured by rotating the input waveguide by $90^{\circ}$. The measured and simulated response of the OMT are shown in Fig. 3. The measured cross-polarisation, return loss, and insertion loss agreed very well with the simulation predictions. The WG13 band of the circular waveguide is indicated by vertical dashed lines. It can be seen that the cross-polarisation and return loss of the OMT, including the coaxial-to-waveguide adaptor and rectangular-tocircular waveguide transition, are about $-58 \mathrm{~dB}$ and $-20 \mathrm{~dB}$ respectively. The difference in insertion loss between the two pairs of opposite probes was found to be less than $0.18 \mathrm{~dB}$ over the design band, as shown in Fig. 4 When incorporated into the C-BASS receiver the OMT will be cooled to $4.2 \mathrm{~K}$, significantly reducing insertion losses. The measurements were repeated for OMT waveguide bodies with $1 \mathrm{~mm}$ longer and shorter backshort lengths, with no significant change in performance.
Figure 2. Measurement setup showing the Device Under Test the coax-to-waveguide adaptor, rectangular-to-circular waveguide transition, waveguide straight, and OMT
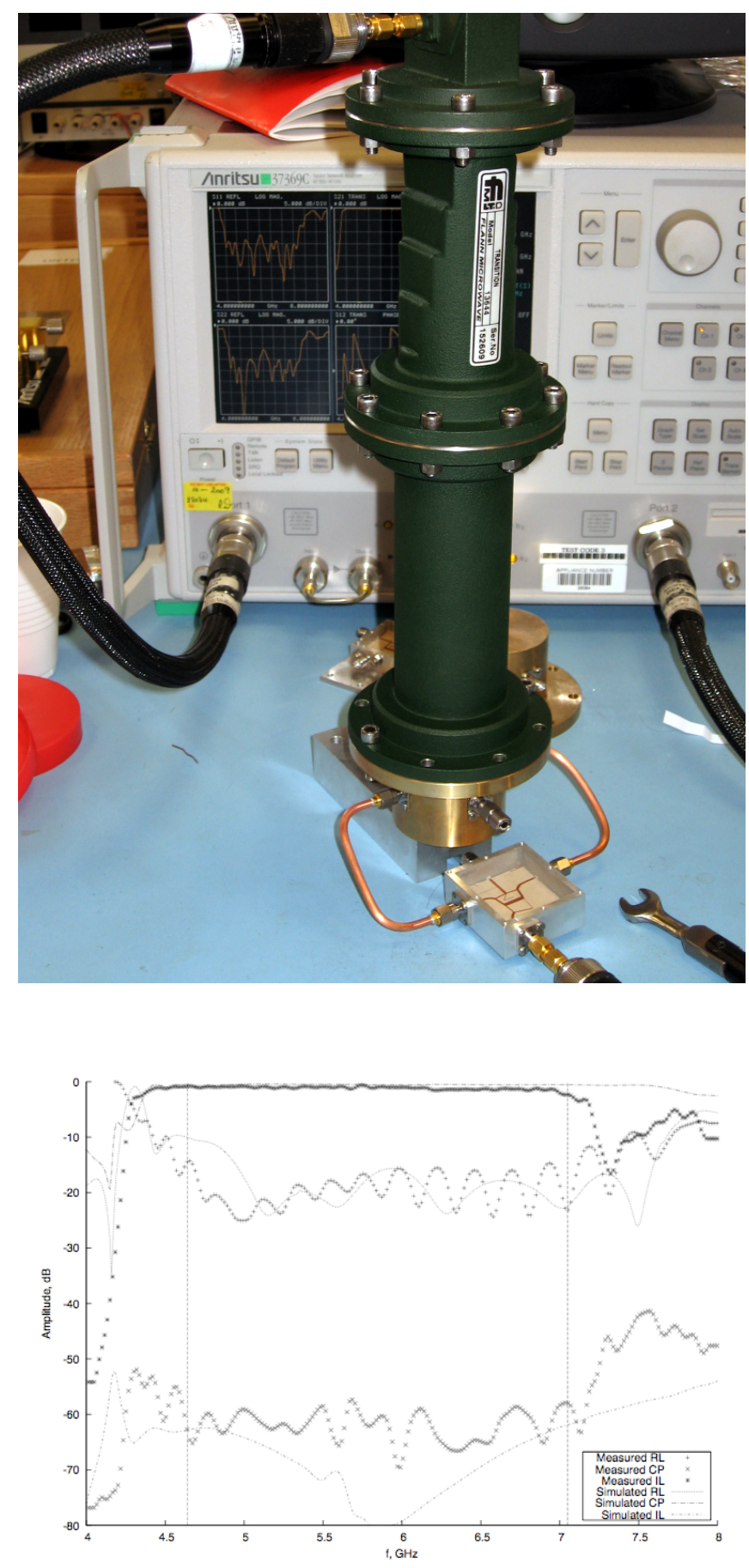

Figure 3. Simulated characteristics of the OMT, and the measured response of the Device Under Test in Fig. 2 The traces plotted are return loss (RL), insertion loss (IL) and cross-polarisation (CP). The rated band of the waveguide (WG13) is indicated by vertical lines

\footnotetext{
1 http://www.zotefoams.com/pages/EN/plastazote.asp

2 http://www.ansoft.com/products/hf/hfss/

3 http://www.ansoft.com/ansoftdesigner/
} 


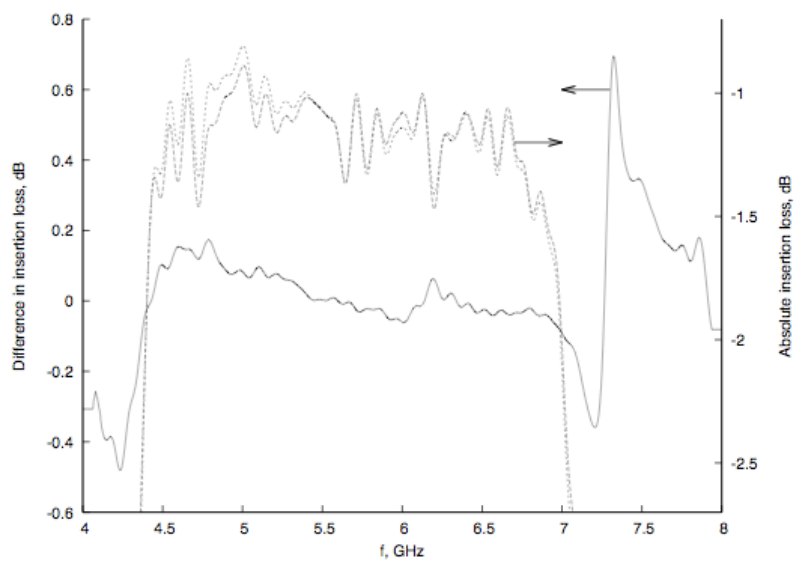

Figure 4. Measured insertion loss of both pairs of opposite probes and the difference between them

\section{CONCLUSION}

We have presented a novel design of a compact planar Cband four probe circular waveguide OMT. We have tested the OMT in the frequency range $4.64 \mathrm{GHz}$ to $7.05 \mathrm{GHz}$ and found excellent agreement between simulations and measured results. We have demonstrated a very low crosspolarisation of less than $-58 \mathrm{~dB}$, a return loss of about $20 \mathrm{~dB}$, and a difference in insertion loss between the orthogonal modes of less than $0.18 \mathrm{~dB}$ over the design band. The OMT is compact, easy to fabricate and yet satisfies the strict requirements of a new generation of radio and $\mathrm{mm}$ cosmological polarisation experiments.

\section{REFERENCES}

Bock, D. (1999), BIMA Memo No. 74.

Engargiola, G. and Plambeck, R. (2003), Review of Scientific Instruments, 74(3), 1380-1382.

Jackson, R. (2001), IEEE Microwave and Wireless Components Letters, 11(12), 483-485.

Knöchel, R. and Mayer, B. (1990), IEEE Microwave Theory and Techniques Symposium Digest.

Taylor, A. (2006), New Astronomy Reviews, 50(11-12), 993-998. 